\title{
Influence of the Sub-peak of Secondary Surface Plasmon Resonance onto the Sensing Performance of a D-shaped Photonic Crystal Fibre Sensor
}

\author{
Suoda Chu, K. Nakkeeran, Senior Member, IEEE, Abdosllam M. Abobaker, Member, IEEE, Sumeet S. Aphale, \\ Senior Member, IEEE, S. Sivabalan, P. Ramesh Babu and K. Senthilnathan
}

\begin{abstract}
In this paper, we design a 6-fold D-shaped photonic crystal fibre sensor based on the surface plasmon resonance (SPR). The coupling between fundamental core mode and three surface plasmonic modes which have different electric filed distributions for analytes of various refractive indices. We observe two different types of SPRs, namely, 'dielectric like' resonance with low-loss peak and 'plasmon like' resonance with high-loss peak, by analyzing the electric field distribution of the fibre modes. Further, we discuss the influence of the secondary SPR over the main SPR which is directly related to the detection performance of the proposed sensor. In order to mitigate the adverse effect of the sub-peak of the secondary SPR on the sensor's dynamic sensing range (DSR), we reduce the thickness of analyte's binding layer from $1500 \mathrm{~nm}$ to $500 \mathrm{~nm}$. Thus, DSR can be extended to $44.4 \%$ from $1.33-1.41$ to $1.33-1.45$ at the cost of a reduced maximum sensitivity from $7900 \mathrm{~nm} / \mathrm{RIU}$ to 5300 nm/RIU. Owning to the simple structure design of the proposed sensor, we envisage that this highly sensitive D-shaped PCFSPR sensor could be developed as a versatile and competitive instrument with a large and flexible refractive index detection range.
\end{abstract}

Index Terms-Sensor, Photonic crystal Fibre, Refractive Index Sensor, Sensitivity, Surface Plasmon Resonance

\section{INTRODUCTION}

$\mathbf{T}$ HE sensing applications based on the optical excitation and detection of the surface plasmon resonance (SPR) phenomenon have been widely studied. It is proven to be a commercially successful technology in the field of medical diagnostics, chemical detection, bio-chemical reaction recognition, food safety control, environment monitoring, etc. The high sensitivity to the change of refractive index of the medium in contact with the surface of thin metal film (typically gold or silver with dielectric), has been widely utilized for those sensing applications [1], [2].

The SPR phenomenon is generally defined as the strong coupling between the electricmagnetic wave and surface plasmon wave at the interface of dielectric and metal [3]. The

Suoda Chu, K. Nakkeeran and Sumeet S. Aphale are with School of Engineering, Fraser Noble Building, University of Aberdeen, Aberdeen AB24 3UE, UK (email: r05sc15@abdn.ac.uk; K.Nakkeeran@abdn.ac.uk; S.Aphale@abdn.ac.uk).

Abdosllam M. Abobaker is with Department of Communications Engineering, College of Electronic Technology, Bani Walid, Libya (email: almahjub11@gmail.com).

S. Sivabalan is with School of Electrical Engineering, VIT University, Vellore-632 014, Tamil Nadu, India (email: ssivabalan@vit.ac.in)

P. Ramesh Babu and K. Senthilnathan are with Department of Physics, School of Advanced Sciences, VIT University, Vellore-632 014, Tamil Nadu, India (email: prameshbabu@vit.ac.in; senthee@gmail.com) development of SPR sensor started from the traditional structure of Kretschmann-Raether prism configuration in 1960. However, this configuration has its own drawbacks such as bulk size, complicated structure design, high cost and low reliability [4]. Eventually, optical fibre evolved as the dielectric medium to overcome the said drawbacks. Due to its design flexibility and compactness [3], the metal coated optical fibres that emerged as an alternative to the prism have been used [5]. As a result, low-cost, highly integrable, and portable optical fibre based SPR sensors have been developed. The most common schemes of SPR fibre sensors include a modified fibre end [6], tapered fibre [7], D-shaped fibre [8], and fiber grating [9].

In recent times, photonic crystal fibre (PCF), also called holey fibre (HF) or micro-structured fibre (MF) has been widely used as a novel class of optical fibre for different sensing applications. Apart from the inherent advantages as a kind of optical fibres, PCFs exhibit their own pros, especially the ability to control its optical characteristics by manipulating the structural parameters of the optical fibre [10]. The PCFSPR based sensors are now widely studied because of their simple and compact probe designed for high sensitivity, robustness, cost effectiveness, fast response, label-free detection [11]. Moreover, it is possible to enhance the sensitivity and the sensing range by optimizing the structural parameters, namely, air holes diameters and the distance between two adjacent air holes.

In the last decade, many metal coating schemes were reported for the PCF-SPR sensor that include completely air hole coating method by Shuai et al in 2012 [12], selectively coated method with the air holes by Yu et al in 2009 [13], the outside metal coating method by Hasan and his research group in the year of 2018 [14] and D-shaped fibre side surface coating by Tian and his research group in 2012 [15]. However, the completely and selectively air holes coating methods are not practically viable besides the challenges of coating the thin metal film homogeneously and uniformly in the inner micrometer-scaled air holes with the existing fabrication technologies. Further, the liquid analyte has to be injected into the air holes by employing those two metal coating methods that increase their complicity and production cost. Although the outside metal coating scheme does not suffer from the above drawbacks, owing its working principle, the sensors based on such methods usually have a very weak surface plasmon resonance which is not observable for sensing. Among these 
metal coating schemes, the D-shaped fibre side surface coating is proven scheme [16]. The hexagonal D-shaped PCF is one of the most frequently used fibre media for common PCF-SPR sensor applications. As the side-polished flat surface is coated with thin metal film that is in direct contact with the liquid analyte, the D-shaped PCF is proposed in this paper for the feasibility of sensor fabrication.

We numerically investigate the sensing performance of a standard fabricable 6-fold hexagonal D-shaped PCF-SPR sensor on a large analyte refractive index range from 1.33 to 1.48. Further, we find that this sensor has a large DSR from 1.33 to 1.41 with a high sensitivity and a linear sensing performance with a liquid layer thickness of $1500 \mathrm{~nm}$. It is worth noting that for lower refractive index analyte $(1.33 \leq$ RI $\leq 1.41$ ), only one 'dielectric-like' SPR with a low-loss peak is observed at $0.6 \mu \mathrm{m}$ wavelength. Hence, one single loss peak is found in the loss spectrum for such detection range. However, for higher refractive index analyte $(1.42 \leq$ $\mathrm{RI} \leq 1.48$ ), two different 'plasmon-like' SPRs with high-loss peaks are observed with two peaks in the same wavelength of $0.6 \mu \mathrm{m}$. Due to the overlap of loss peak and the existence of the sub-peak, as a single-peak detection device, the sensing performance is literally affected and this drawback makes this RI range undetectable. Moreover, the two resonance coupling mechanisms of 'dielectric like' SPR and 'plasmon like' SPR for low and high RI analyte ranges are different. The wavelength interrogation method is applied for SPR optical fibre sensing to evaluate the sensitivity of the proposed sensor due to the availability of affordable small optical spectrum analyzers [17]. The simulation results indicate that the highest sensitivity for an RI range of 1.33-1.41 is up to $7900 \mathrm{~nm} / \mathrm{RIU}$. By reducing the liquid layer thickness to $500 \mathrm{~nm}$, the DSR can be extended to 1.33-1.45. But on the downside, the maximum sensitivity reduces to $5300 \mathrm{~nm} / \mathrm{RIU}$.

\section{Geometric Structure AND Numerical MODELLING}

The schematic of the proposed sensor is shown in Fig. 1. It comprises of 4 layers of air holes arranged in a six-fold hexagonal PCF structure of air hole diameter, $d=1.2 \mu \mathrm{m}$ in a solid core. The distance between two holes (pitch), $\Lambda$, is 2.5 $\mu \mathrm{m}$ and the radius of the whole sensor is set as $11 \mu \mathrm{m}$. An open D-shaped analyte channel is designed at the top part of the fibre cross-section so that the analyte can be infused in the channel. The height of the D-shaped channel, $d_{a}$, is $8 \mu \mathrm{m}$. An uniform nano-scale gold metal film is deposited on the flat side-polished surface with its layer thickness of $t_{\mathrm{Au}}=45 \mathrm{~nm}$ for surface plasmon polaritons generation. The thickness of liquid analyte layer in the D-shaped channel is kept at 1500 $\mathrm{nm}$.

In this structure, fused silica is chosen as the cladding material and the wavelength dependence of the refractive index of the silica glass is calculated by the Sellmeier equation [18]. The dielectric constant of the thin gold film is calculated using the Drude model that is characterized in [19].

The proposed structure can be fabricated using the stateof-the-art technique of stack-and-draw [20] and side polishing

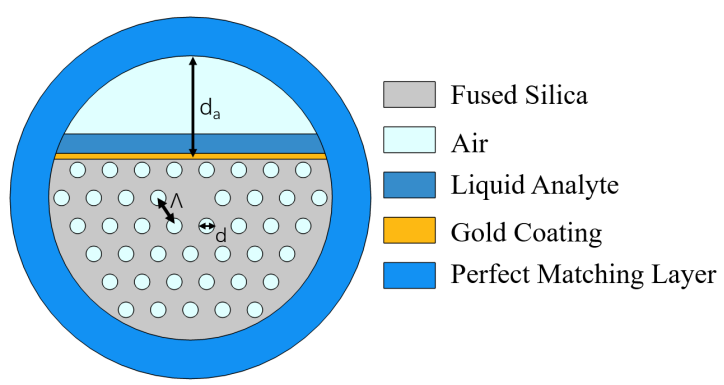

Fig. 1. Cross-section of the proposed six-fold D-shaped hexagonal PCF-SPR sensor.

methods [21]. The fabrication process of proposed sensor is also based on D-shaped side-channel PCF fabrication which has been successfully reported by Chen $e t$ al. They used two steps stack-and-draw process and the channel was created by removing the top of the holy lattice cladding as illustrated in Fig. 2 [16]. During the fibre drawing process, the pressure, vacuum and the stability of drawing tower need to be well controlled to maintain the structure of the PCF [22]. In order to achieve a D-shaped channel, the side polishing method can be used to remove the top part. The rugged surface of the D-shaped upper side can be processed by a focused highpower laser beam through the cavity to achieve flat surface of the channel. Finally, a thin gold layer is coated on the sensing surface with a chemical deposition method, described by Jonathan Boehm [23].

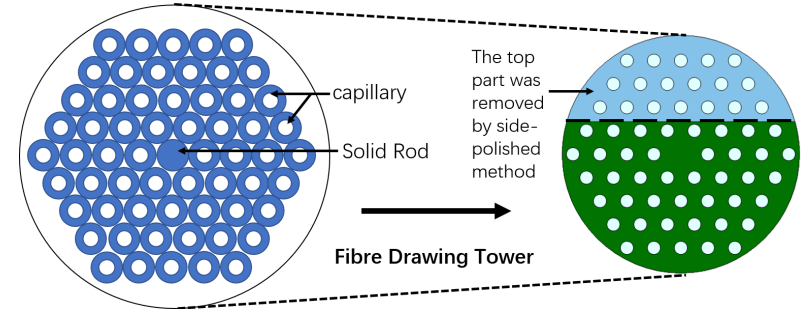

Fig. 2. Stacked preform of the proposed PCF and side polishing method for the top part removal.

To numerically investigate the sensing performance of this sensor, FEM is used to find the effective refractive indices through COMSOL Multiphysics software. A perfectly matched layer (PML) is considered as the boundary condition for the outside edges to absorb the radiated light energy for the simulation to mimic the real situation [24].

\section{Analysis of Modes}

For any SPR based sensor, it is well known that the surface plasmon mode (PM) is generated and coupled with the main core-guided fundamental mode (FM) at a particular resonant wavelength [25]. In essence, there occurs a transfer of energy from FM to PM. Thus, at the resonance wavelength, the loss spectrum exhibits a sharp resonance-peak characteristic [17].

Simulations results of the designed PCF-SPR sensor for the formation of the PM are shown in Figs. 3. The refractive index of the liquid analyte, $n_{a}$, is 1.38 in the lower RI range (1.33 $\leq \mathrm{RI} \leq 1.41$ ). Figures 3 illustrate the distribution of the light of the FM as well as the PM of the proposed sensor at various resonance wavelengths of $600 \mathrm{~nm}, 690 \mathrm{~nm}$ and and $770 \mathrm{~nm}$. 

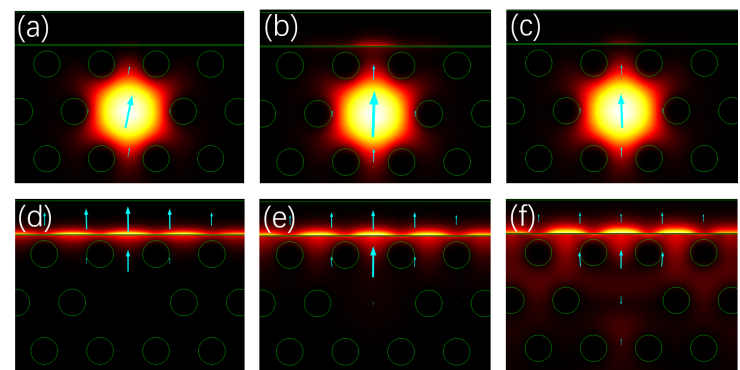

Fig. 3. Distribution of light in the cross-section of D-shaped PCF-SPR sensor for different wavelengths for the analyte RI of $n_{a}=1.38$. (a) and (d) are the FM and PM at $600 \mathrm{~nm}$ (shorter wavelength with respect to the resonance). (b) and (e) are the FM and PM at resonant wavelength of $690 \mathrm{~nm}$. (c) and (f) are the FM and PM at $770 \mathrm{~nm}$ (longer wavelength with respect to the resonance).The arrows indicate the direction of the electric field.

Here, the arrows indicate the direction of the electric field. Figures 3(d), 3(e) and 3(f) show the formation of the PM for all the three wavelengths. In Fig. 3(b), a weak surface plasmon resonance can be observed at the inference between the thin gold film and the dielectric. Figs. 3(a) to 3(c) clearly demonstrate the complete mechanism of SPR wherein a part of light energy of FM leaks into the gold film sensing area as the light signal of PM.

Figure 4 shows the confinement loss spectrum (solid curve), dispersion relations of the FM (dashed curve) and PM (dotdashed curve) for the D-shaped hexagonal PCF-SPR sensor for an analyte of RI of 1.38. Here, the confinement loss is calculated in $\mathrm{dB} / \mathrm{cm}$ using the relation [26],

$$
\alpha_{\text {loss }}=8.686 \times \frac{2 \pi}{\lambda} \Im\left(n_{\text {eff }}\right) \times 10^{4},
$$

where $\Im\left(n_{\text {eff }}\right)$ is the imaginary part of the effective refractive index of the FM. As it is illustrated in Fig. 4, a sharp and deep single resonance peak is observed at the resonance wavelength of $690 \mathrm{~nm}$.

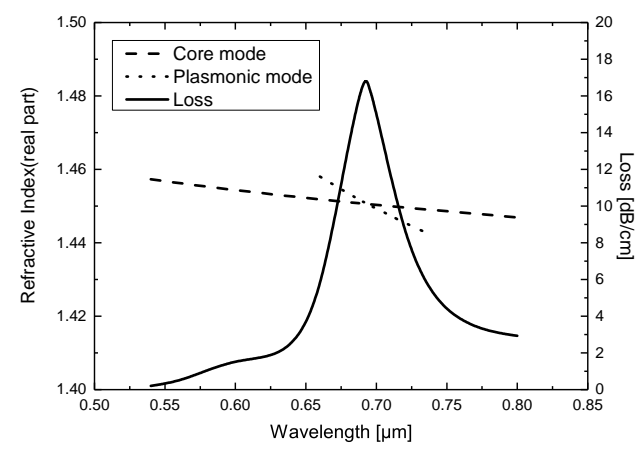

Fig. 4. The confinement loss and the dispersion relations of FM and PM for the analyte RI of $n_{a}=1.38$.

However, as shown in Fig. 5, two resonance peaks are observed in the confinement loss spectrum at resonance wavelengths of $1337.5 \mathrm{~nm}$ and $1515 \mathrm{~nm}$ for an higher refractive index analyte of 1.46. The surface plasmon modes in these two SPRs exhibit different patterns as depicted in Figs. 6 and Figs. 7 when compared to what has been illustrated in Figs. 3. The electric field distributions of FM and PM are shown in Figs. 6 and Figs. 7 for the first and second resonance wavelengths, respectively, that include both shorter and longer wavelengths.

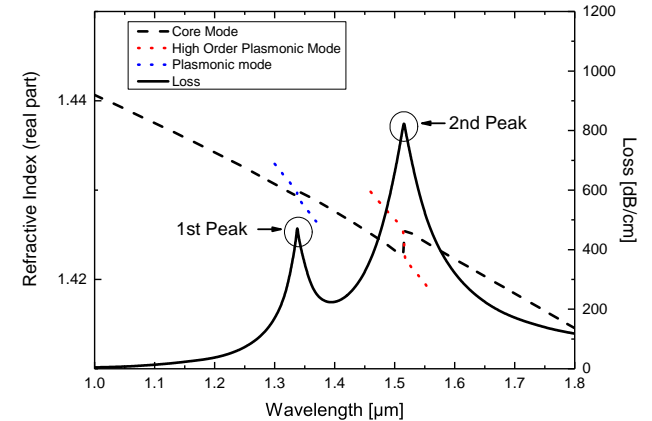

Fig. 5. The confinement loss and the dispersion relations of FM and PM for the analyte RI of $n_{a}=1.46$.
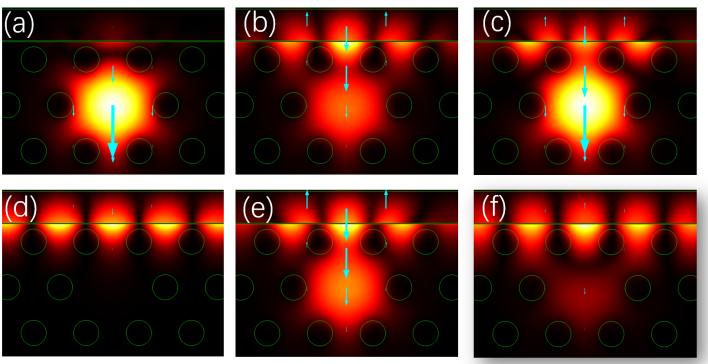

Fig. 6. Distribution of light in the cross-section of D-shaped PCF-SPR sensor for different wavelengths for the analyte RI of $n_{a}=1.46$. (a) and (d) are the FM and PM at $1100 \mathrm{~nm}$ (shorter wavelength with respect to the resonance). (b) and (e) are the FM and PM at resonant wavelength of $1337.5 \mathrm{~nm}$. (c) and (f) are the FM and PM at $1400 \mathrm{~nm}$ (longer wavelength with respect to the resonance). The arrows indicate the direction of the electric field.

As is evident in Figs. 3(b), Figs. 6(b) and Figs. 7(b), the electric field distribution of SPR is differen for different resonance wavelengths. We note that the electric field of 'dielectric-like' resonance is mainly confined in the solid core area in the case of analyte of lower RI. In this case, the photons from the fundamental mode propagate through the thin gold film and get coupled with the coherent delocalized electrons of the gold film in the form of surface plasmons that are generated at the upper boundary between thin gold film and the analyte layer. Here, the strength of sensing signal is low but detectable. At its resonance wavelength, the electric field pattern of surface plasmon does not change apprecialbly as illustrated in Figs. 3(e) when compared to Figs. 3(d) and (f).

On the other hand, for higher RI analyte, the electric fields of two 'plasmon-like' resonances are mainly concentrated at the gold film surface with extremely high loss, as shown in
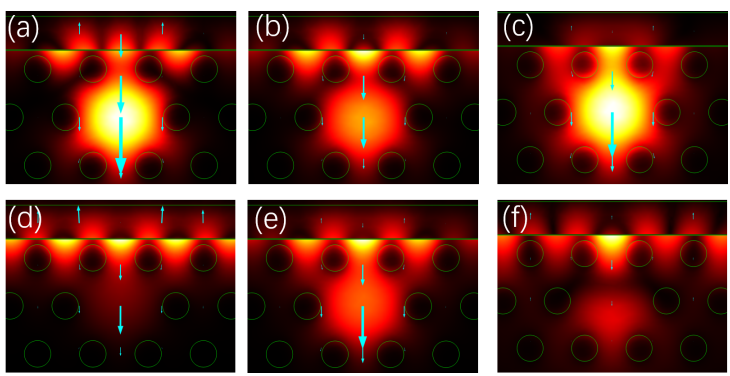

Fig. 7. Distribution of light in the cross-section of D-shaped PCF-SPR sensor for different wavelengths for the analyte RI of $n_{a}=1.46$. (a) and (d) are the FM and PM at $1400 \mathrm{~nm}$ (shorter wavelength with respect to the resonance). (b) and (e) are the FM and PM at resonant wavelength of $1515 \mathrm{~nm}$. (c) and (f) are the FM and PM at $1800 \mathrm{~nm}$ (longer wavelength with respect to the resonance). The arrows indicate the direction of the electric field. 
Figs. 6(b) and Figs. 7(b). On comparing the electric field patterns of the surface plasmons displayed in Figs. 6(c),(e),(f) and Figs. 7(c),(e),(f), we find that the surface plasmon can not only absorb a small portion of leaked light at resonance, but also radiates part of it to core to form a quasi-core distribution at resonance wavelength. It is to be noted that this absorption and radiation in both shorter and longer wavelengths are not as strong as that of resonance wavelength. Moreover, the strongest coupling/absorption happens at the lower boundary of gold film with a weak surface plasmon generated on the upper gold film surface which has the same phase [Figs. 6(d)] or phase shift [Figs. 7(d)] with the lower boundary surface plasmon.

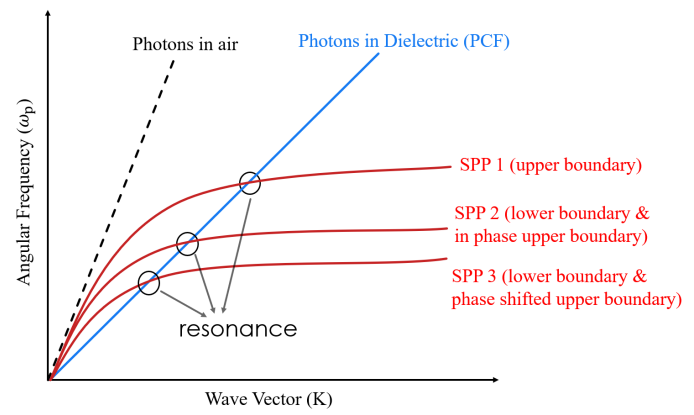

Fig. 8. Dispersion relations of air (dashed line), fiber medium (blue) and upper and lower surfaces of thin gold film (red). The circles indicate occurrence of SPR in three different circumstances.

Three surface plasmon resonances with different electric field distributions, as illustrated in Figs. 8, can be found by the coulpings that happen between different surface plasmon polaritons (SPPs) and the photons. Among them, the SPP 1 describes the surface plasmon mode of Figs. 3(d), SPP 2 corresponds to the surface plasmon mode of Figs. 6(d) and SPP 3 is for the surface plasmon mode of Figs. 7(d). This phenomenon can be explained by the high reflectance of gold film at longer wavelength which is related to metallic reflectance and the increased RI of the analyte. A standard reflectance spectrum of gold shown in Fig. 9 is related to the RI by the following equation [27]:

$$
R=\frac{(n-1)^{2}+k^{2}}{(n+1)^{2}+k^{2}},
$$

where $R, n$ and $k$ represent the reflectance, real part and imaginary parts of refractive index, respectively. It is obvious that, at higher wavelengths, the gold film will exhibit high reflectance characteristic and vice versa.

For lower RI analytes, as their resonance wavelengths are normally distributed in the shorter wavelengths region, the gold film would have a lower reflectivity. Hence, most of the leaked light energy gets transmitted through the gold film and excites the SPR together with the delocalized electrons at the upper surface of the gold film. However, the SPPs at the lower surface of gold are too weak to observe. Moreover, as the RI of analyte is lower than that of fibre silica, light gets confined well in a fibre medium [28] and hence the confinement loss of this sensor is very low. Therefore, a weak SPR takes place at the upper surface of gold film. However, for higher RI analytes, a larger portion of light leaks from FM to PM and it results in

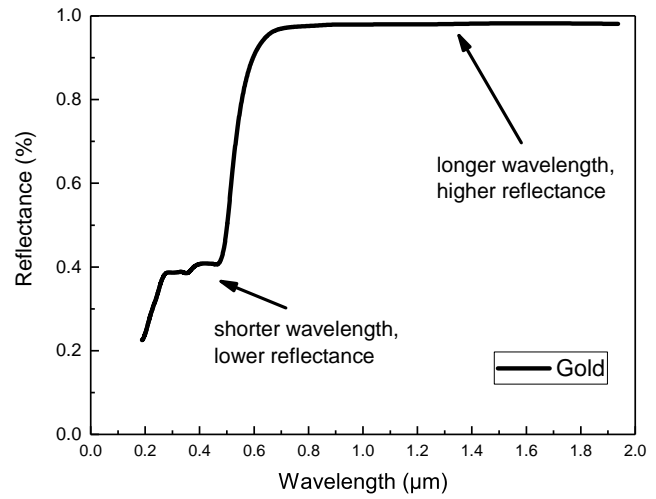

Fig. 9. Standard reflectance spectrum for gold

high confinement loss as seen in Fig. 5. Besides, the leaked light energy cannot easily transmit through the gold film as the reflectivity of the metal is higher at longer wavelength. Unlike the case of SPPs at the lower wavelength, most of the leaked photons get coupled with the delocalized electrons at the lower surface of gold film and only a small portion of them can get transmitted through the gold film. As a result, the gold film has stronger surface plasmon at lower surface and weaker surface plasmon at the upper surface.

At the resonance wavelength, due to maximum confinement loss, the energy absorption of surface plasmon turns out to be a maximum. Thus, the leaked photons can not fully couple with electrons from metal layer. The rest uncoupled photons from leaked light energy will be continually absorbed by the SPP. However, the excess SPP energy is transformed into radiation [29]. The radiation energy from SPP is reflected by the gold film back into the fibre core. However, the radiated photons that have the same dispersion relations with surface plasmon mode will gather and form a quasi-core mode in the core area. The surface plasmon modes shown in Figs. 6(e) and Figs. 7(e) turn out to be hybrid modes of a surface-plasmon mode for absorption at gold film and a quasi-core mode for radiation at core area.

Moreover, in Fig. 7(d), the surface plasmons are formed by the delocalized electrons at the lower boundary of gold film and hence weak surface plasmons are formed with a phase shift of $\pi$ on the upper surface. Although a strong coupling between fundamental mode and hybrid surface plasmon occurs at the lower boundary of gold film, the weak surface plasmon on upper side of gold film still gets involved in the resonance. This results in 'plasmon-like' SPR which is also sensitive to the RI changes of the analyte. However, due to the increasing reflectance of gold in longer wavelength, the sensing ability based on 'plasmon-like' SPR will decrease for higher RI analyte.

Thus, for a large range of RI analytes, there are two different types of SPRs, namely, the 'dielectric-like' and the 'plasmonlike', respectively due to different resonance wavelengths. Further, they are also known as 'incomplete coupling' and 'complete coupling' [12] [30] [31] [32]. We note that the phase matching conditions for them are found to be different. For lower RI of analyte, as its resonance wavelength is usually located at shorter wavelength region, the 'dielectric-like' SPR occurs at upper boundary of gold film surface due to the the 
phase matching condition which demands that the real parts of effective refractive indices ( $\left.n_{\text {eff }}\right)$ of FM and PM be equal. However, for the analytes of higher RIs, as the resonance wavelengths are usually located at longer wavelength region, the 'plasmon-like' SPR occurs at lower surface of gold film. As discussed above, the hybrid surface plasmon mode of 'plasmon-like' SPR actually combination of absorption and radiation. In 2008, Zhang et al have noticed that there would be two different coupling phenomenona between modes and successfully proved them [33]. Based on their coupled-mode theory, in any SPR, the parameters $\kappa$ and $\delta_{\mathrm{i}}$ represent modecoupling strength, which is related to the real part of $n_{\mathrm{eff}}$, and mode-absorption strength, which is related to the imaginary part of $n_{\text {eff }}$, respectively. For 'dielectric-like' SPR, as there is only mode coupling between fundamental mode and upper surface mode, the $\kappa$ is always greater than $\delta_{\mathrm{i}}$. Hence, the incomplete coupling takes place under the condition with equal real parts of two modes. For 'plasmon-like' SPR, the mode-coupling strength $\kappa$ is defined between fundamental core mode and hybrid surface plasmon mode. However, the mode-absorption strength $\delta_{\mathrm{i}}$ is related to the surface plasmon mode absorption and the radiant energy. In this situation, $\delta_{\mathrm{i}}$ is greater than $\kappa$. Therefore, a complete coupling occurs when the imaginary parts of $n_{\text {eff }}$ of fundamental core modes are equal to the $n_{\text {eff }}$ of hybrid surface plasmons. To the best of our knowledge, this is the first time to reveal and explain these phenomena by analysing the electric distribution of different fibre modes. Also, this is the first time to observe the existence of surface plasmon resonance at different surfaces of gold film.

\section{SENSING PERformANCE}

In this section, we analyze the performance of the proposed sensor. For a better performance of a refractive index based SPR fibre sensor, the energy transferred to the PM needs to be extremely sensitive to the RI changes of the aqueous analyte [34]. When there are small RI changes in the analyte due to chemical/biochemical interactions, the real part of the $n_{\text {eff }}$ of the PM undergoes a shift its position with respect to wavelength. As a consequence, the resonant wavelength occurring at the phase matching condition will also have a significant shift.

One can observe single resonance peaks from the loss spectra of Fig. 10(a) for different analytes of RI ranging from 1.33 to 1.41 within the wavelength range from 0.5 to 1.0 $\mu \mathrm{m}$. Here, the confinement loss increases with the increase in RI of the analyte. It should be noted that the loss peaks for $\mathrm{RI}=1.42$ and 1.43 become more and more blunt and broad due to increase in confinement loss and the existence of a secondary SPR caused by FM and hybrid PM in the longer wavelength. As a result, the resonance peak can hardly be obtained and observed by a spectrometer. However, dual resonance peaks are observed in Fig. 10(b) for a wavelength range from 0.9 to $1.8 \mu \mathrm{m}$ for the analytes of RI varying from 1.42 to 1.48 . Due to the existence of SPR between fundamental core mode and hybrid surface plasmon, a sub-peak is observed in the confinement loss spectra. As the secondary SPR always occurs at a longer wavelength, it is a 'plasmon-like' SPR with very high loss. Although the sub-peak is still sensitive to the RI changes of the analyte, the overlap of resonance peaks causes an adverse effect on sensing performance. Thus, in the detection process, dual-peak characteristics will cause errors in the measurement within the certain sensing bandwidth. It is clear that the uniqueness and accuracy of single peak measurement cannot be guaranteed by the proposed sensor for analytes whose RI is greater than 1.41 due to the overlap of two resonance peaks. This is a limitation on detection range and sensing performance of the proposed sensor.
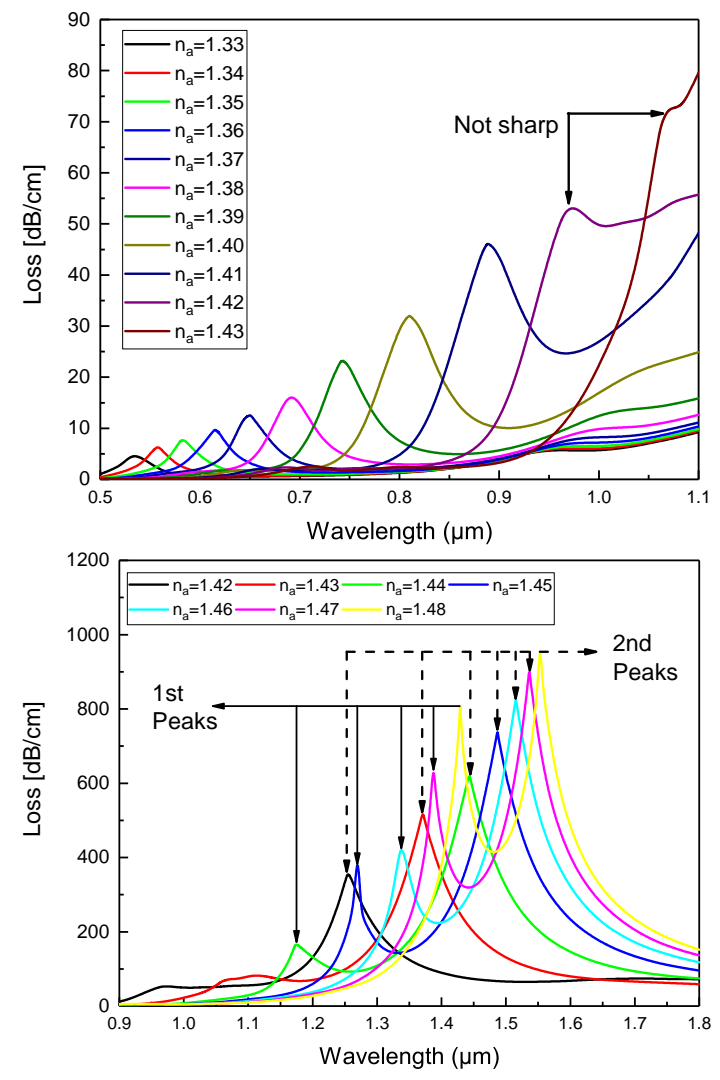

Fig. 10. The loss spectra for the analyte refractive index $\left(n_{a}\right)$ varying from (a) 1.33 to 1.43 in steps of 0.01 and (b) 1.42 to 1.48 in a step of 0.01 .

By introducing the wavelength interrogation method, the sensitivity, $S_{\lambda}(\lambda)$, of the sensor can be calculated using the expression [2],

$$
S_{\lambda}(\lambda)=\frac{\Delta \lambda_{\text {peak }}}{\Delta n_{a}},
$$

where $\Delta \lambda_{\text {peak }}$ is the resonant wavelength shift and $\Delta n_{a}$ is the analyte's refractive index difference. For example, in Fig. 10(a), the wavelength shift between the confinement loss peaks for RI 1.40 and 1.41 is $79 \mathrm{~nm}$. Hence, the sensitivity of the PCF-SPR sensor for the analyte RI changing from 1.40 to 1.41 is calculated to be $7900 \mathrm{~nm} / \mathrm{RIU}$.

Table I reports the resonant wavelengths and the corresponding sensitivities of the PCF-SPR sensor for various detectable analytes'. The sensor exhibits a maximum sensitivity of $7900 \mathrm{~nm} / \mathrm{RIU}$ under 'dielectric-like' SPR with a low loss for analyte's RI ranging from 1.33 to 1.41 and the maximum resolution is as high as $1.26 \times 10^{-5}$ RIU for a $0.1 \mathrm{~nm}$ peak-wavelength resolution of the instrument for precise and accurate detection. 
TABLE I

SENSITIVITY DATA OF THE D-SHAPED PCF-SPR SENSOR (ANALYTE RANGES FROM 1.33 TO $1.41\left[T_{\mathrm{a}}\right.$ IS $1500 \mathrm{NM}$ ])

\begin{tabular}{lcccc}
$\begin{array}{l}\text { Analyte } \\
\text { Refractive } \\
\text { Index }\end{array}$ & $\begin{array}{c}\text { Resonant } \\
\text { Wavelength } \\
(\mathrm{nm})\end{array}$ & $\begin{array}{c}\text { Peak } \\
\text { Loss } \\
(\mathrm{dB} / \mathrm{cm})\end{array}$ & $\begin{array}{c}\text { FWHM Loss } \\
\text { Bandwidth } \\
(\mathrm{nm})\end{array}$ & $\begin{array}{c}\text { Sensitivity } \\
(\mathrm{nm} / \mathrm{RIU})\end{array}$ \\
\hline & & & & \\
1.33 & 530 & 4.696 & 21.0 & - \\
1.34 & 557 & 6.263 & 29.8 & 2700 \\
1.35 & 585 & 7.676 & 32.3 & 2800 \\
1.36 & 614 & 9.745 & 37.7 & 2900 \\
1.37 & 650 & 12.59 & 42.5 & 3600 \\
1.38 & 690 & 16.76 & 49.7 & 4000 \\
1.39 & 744 & 23.19 & 50.4 & 5400 \\
1.40 & 810 & 32.00 & 63.8 & 6600 \\
1.41 & 889 & 46.22 & 100.0 & 7900
\end{tabular}

There are different types of analytes that can be detected using the proposed sensor as long as the surface plasmon mode is created for the sensing range of refractive indices (1.33 to 1.41) of the samples that are of interest, such as water (1.33), glucose solution in water (1.34 to 1.36 , depending on the concentration percentage) or ethyl alcohol (1.36). The PCF-SPR sensors now are also widely used as bio-sensors for detecting and monitoring the aqueous based immobilise biomolecules in antigen-antibody interaction binding process [11]. The local refractive index of analyte binding-layer changes as the analytes selectively bind to the functionalized surface [35]. In order to reduce the substantial impact from the sub-peak of SPR to the DSR, one has to change the binding layer thickness of analyte ( $\left.T_{\text {binding }}\right)$. As a result, its sensing range increases at the cost of sensitivity. Therefore, it is of paramount importance to analyze the performance of sensor for various binding-layer thicknesses of analytes. By decreasing the $T_{\text {binding }}$ to $500 \mathrm{~nm}$, it is possible to extend the detectable RI sensing range from 1.33-1.41 to 1.33-1.45 with a growth rate of $45 \%$. Figure 11 shows the confinement loss spectra for the analyte RI varying from 1.33 to 1.45 when $T_{\text {binding }}$ is $500 \mathrm{~nm}$ and the calculated sensitivity and FWHM loss bandwidth are summarised in Table II. It is to be noted that the maximum sensitivity sensitivity of the thicker binding layer $(1500 \mathrm{~nm})$ reduces from $7900 \mathrm{~nm} / \mathrm{RIU}$ to $5300 \mathrm{~nm} / \mathrm{RIU}$ for a thinner analyte binding layer of $500 \mathrm{~nm}$. Moreover, the maximum sensitivity is found at the analyte RI of 1.41 which is same as the sensing results of the one with thicker binding layer, and a trend of sensitivity drop can be seen for the higher analyte RIs. Hence, the proposed sensor provides a better sensitivity within the analyte RI range of 1.33 to 1.41 when the analyte's binding thickness is $1500 \mathrm{~nm}$. However, the detectable sensing range can be extended to $1.33-1.45$ by decreasing the analyte's binding thickness to $500 \mathrm{~nm}$ at the cost of reduced sensitivity. With an extended sensing DSR, it is possible to detect different kinds of analytes such as coconut oil (1.43), olive oil (1.44) and so on.

The peak losses and FWHM bandwidths of the loss spectra corresponding to different RI of the analyte when the analyte binding thickness $T_{\text {binding }}$ is set to $500 \mathrm{~nm}$ or $1500 \mathrm{~nm}$ are shown in Fig. 12(a) and (b). We can see clearly that the peak loss and FWHM bandwidth increase with the increase in RI of analyte for two different binding layer thickness of the analyte.
Here, the tremendous increase in FWHM bandwidth is caused by the secondary surface plasmon resonance as it blunts the first resonance peak. Loss resonant wavelengths for different binding layer thicknesses are also plotted in Fig. 12(c) and (d) with straight line fitting. The loss resonant wavelength values show a linearly increase trend with the increasing values of the refractive index of the analyte. The fitting lines for the analyte's binding layer thicknesses, $500 \mathrm{~nm}$ and 1500 $\mathrm{nm}$ result in the R-squared values of 0.98483 and 0.95977 , respectively. In this case, $\mathrm{R}$-squared values close to 1 indicate that the proposed sensor has excellent linear characteristics on the sensing performance. Further, it is also clear that the thinner analyte binding layer has a better linearity than the one with thicker binding layer. Using the sensitivity (S) value and the FWHM bandwidths data, we can calculate the figure of merit (FOM) [36] of the designed sensor as,

$$
\mathrm{FOM}=\frac{\mathrm{S}(\mu \mathrm{m} / \mathrm{RIU})}{\text { FWHM Bandwidth }(\mu \mathrm{m})} .
$$

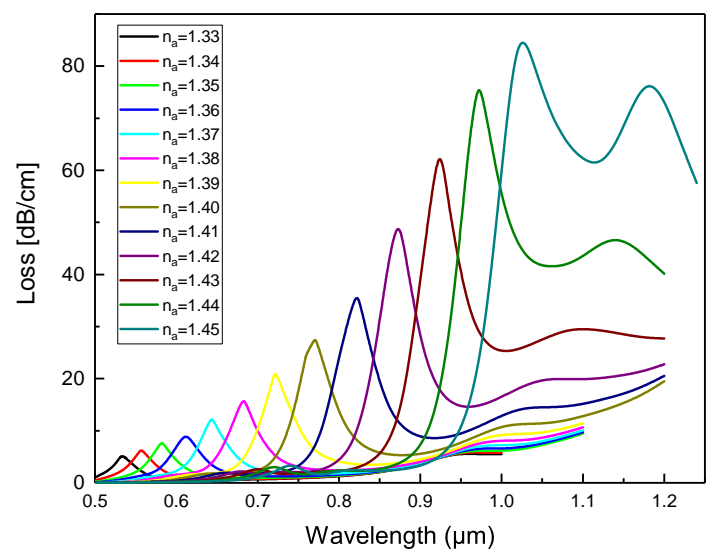

Fig. 11. The loss spectra for the analyte refractive index $\left(n_{a}\right)$ varying from 1.33 to 1.45 in a step of 0.01 .

TABLE II

SENSITIVITY DATA OF THE D-SHAPED PCF-SPR SENSOR (ANALYTE RANGES FROM 1.33 TO 1.45 [ $T_{\text {binding }}$ IS $500 \mathrm{NM}$ ])

\begin{tabular}{lcccc}
$\begin{array}{l}\text { Analyte } \\
\text { Refractive } \\
\text { Index }\end{array}$ & $\begin{array}{c}\text { Resonant } \\
\text { Wavelength } \\
(\mathrm{nm})\end{array}$ & $\begin{array}{c}\text { Peak } \\
\text { Loss } \\
(\mathrm{dB} / \mathrm{cm})\end{array}$ & $\begin{array}{c}\text { FWHM Loss } \\
\text { Bandwidth } \\
(\mathrm{nm})\end{array}$ & $\begin{array}{c}\text { Sensitivity } \\
(\mathrm{nm} / \mathrm{RIU})\end{array}$ \\
\hline & & & & \\
1.33 & 535 & 5.142 & 26.6 & - \\
1.34 & 557 & 6.239 & 29.0 & 2200 \\
1.35 & 582 & 7.666 & 29.3 & 2500 \\
1.36 & 611 & 9.587 & 32.2 & 2900 \\
1.37 & 644 & 12.22 & 33.5 & 3300 \\
1.38 & 680 & 15.87 & 37.8 & 3600 \\
1.39 & 723 & 21.02 & 38.6 & 4300 \\
1.40 & 770 & 27.65 & 43.9 & 4700 \\
1.41 & 820 & 36.49 & 44.3 & 5000 \\
1.42 & 873 & 49.27 & 45.6 & 5300 \\
1.43 & 923 & 62.73 & 48.2 & 5000 \\
1.44 & 972 & 75.93 & 54.9 & 4900 \\
1.45 & 1020 & 89.61 & 124.0 & 4800
\end{tabular}

From Fig. 12(e), it should be noted that the corresponding FOM of the sensor will be degraded sharply as the FWHM bandwidth is broadened due to the the secondary resonance at the longer wavelength. The average FOM obtained for 

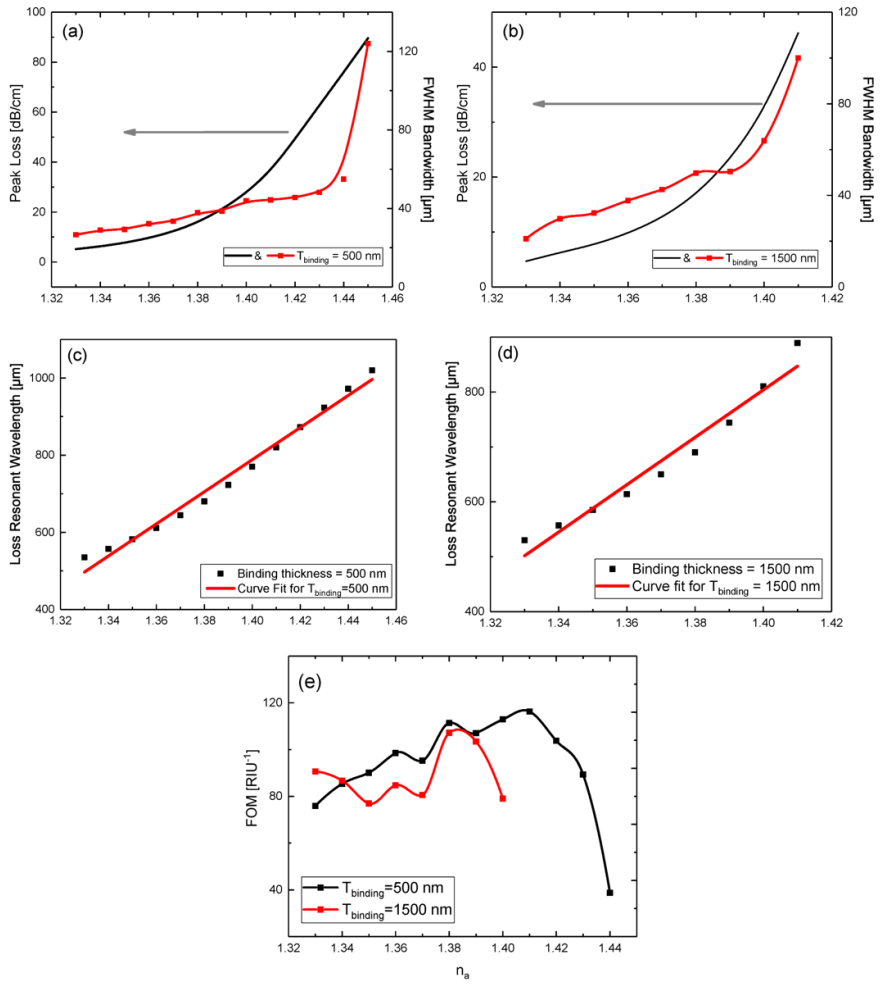

Fig. 12. (a) \& (b) Peak loss and FWHM bandwidth for various analytes' RI (c) \& (d) Loss resonant wavelengths and straight lines fit, (e) FOM versus analytes' RI, for different analyte binding thicknesses $\mathrm{T}_{\mathrm{binding}}=500 \mathrm{~nm}$ and $1500 \mathrm{~nm}$, respectively.

analyte's binding thickness of $500 \mathrm{~nm}$ and $1500 \mathrm{~nm}$ are respectively calculated to be 88.6 /RIU and 93.7 /RIU and these values are higher than the FOM reported in [11].

\section{EFfect of VARiations in Structure Parameters}

In this section, we analyze the sensing performance of the proposed sensor by varying the structural parameters, namely, air holes distance $(\Lambda)$, air holes diameter (d) and thickness of metal layer $\left(t_{\mathrm{au}}\right)$. Figures 13 show the variation of confinement loss against the different air hole diameters of $1.1,1.2$ and $1.3 \mu \mathrm{m}$, different pitch distances of $2.4 \mu \mathrm{m}$, $2.5 \mu \mathrm{m}$ and $2.6 \mu \mathrm{m}$, different gold layer thicknesses of 30 $\mathrm{nm}, 45 \mathrm{~nm}$ and $60 \mathrm{~nm}$ for the analyte RI values of 1.38 and 1.39 and the analyte binding layer thickness is $500 \mathrm{~nm}$. In Fig. 13(a), it can be seen that when the air hole diameters decrease, the resonant wavelength undergoes blueshift and the loss is decreases as well. The resonance peak wavelength shifts for different air hole diameters are calculated as $44 \mathrm{~nm}$ $(1.1 \mu \mathrm{m}), 43 \mathrm{~nm}(1.2 \mu \mathrm{m})$ and $39 \mathrm{~nm}(1.3 \mu \mathrm{m})$ with their corresponding sensitivities of $4400 \mathrm{~nm} / \mathrm{RIU}, 4300 \mathrm{~nm} / \mathrm{RIU}$ and $3900 \mathrm{~nm} / \mathrm{RIU}$, respectively. The sensitivity is slightly increased with the decrease of air holes diameter.

As it is illustrated in Fig. 13(b), the resonant wavelength shifts are $45 \mathrm{~nm}(2.4 \mu \mathrm{m}), 43 \mathrm{~nm}(2.5 \mu \mathrm{m})$ and $39 \mathrm{~nm}(2.6 \mu \mathrm{m})$ for different air hole distances. It is obvious that the sensitivity calculated from the resonant wavelength shift increases with the decrease of pitch. The resonant peak wavelength will shift to longer wavelength with the decrease of pitch value but the loss decreases. In Fig. 13(c), the resonant wavelength shifts for three different gold coating thickness of $30 \mathrm{~nm}, 45 \mathrm{~nm}, 60$
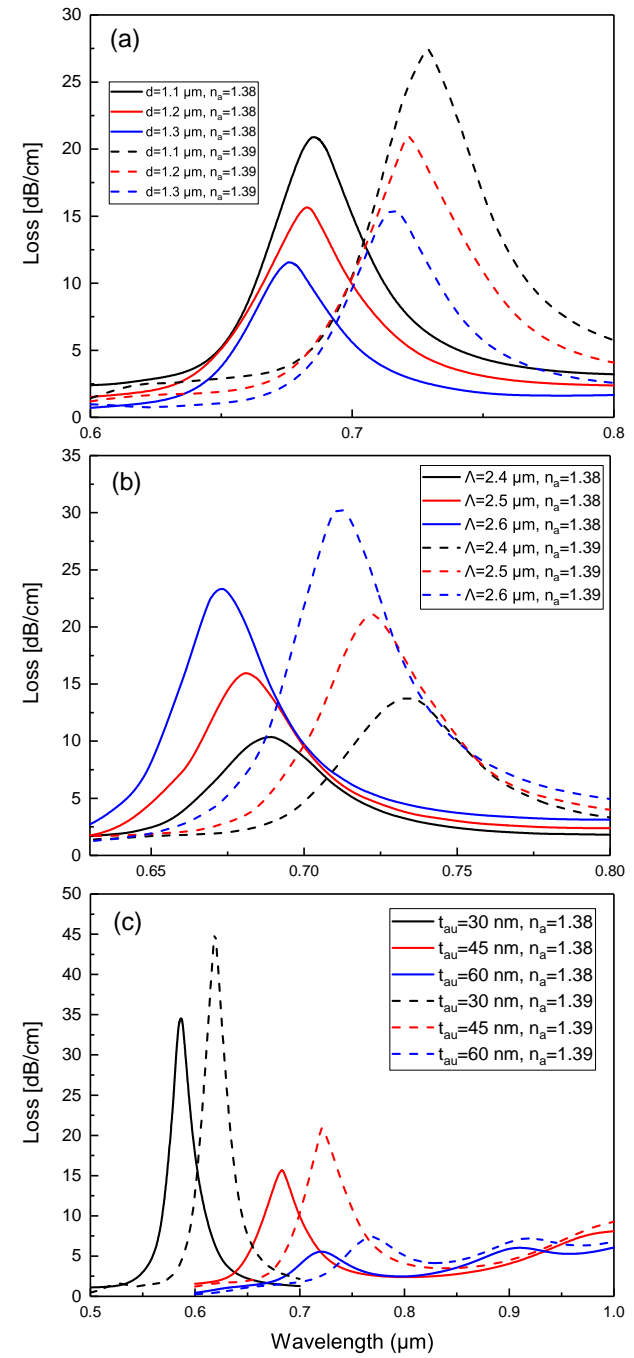

Fig. 13. Variation of the loss spectra for (a) Three different air holes diameters, $1.1 \mu \mathrm{m}, 1.2 \mu \mathrm{m}, 1 . \mu \mathrm{m}$, (b) Three different pitches of $2.4 \mu \mathrm{m}, 2.5 \mu \mathrm{m}, 2.6$ $\mu \mathrm{m}$ (c) Three different thin gold coating thicknesses of $30 \mathrm{~nm}, 45 \mathrm{~nm}, 60 \mathrm{~nm}$ with a analyte binding layer thickness of $500 \mathrm{~nm}$ for RI of 1.38 and 1.39 .

$\mathrm{nm}$ are calculated as $32 \mathrm{~nm}, 43 \mathrm{~nm}$ and $47 \mathrm{~nm}$, respectively. Although the increase of gold coating thickness results in a reduction on the sensitivity of the proposed sensor, the loss spectrum curve becomes sharper with the higher loss so that the resonant peak can be found more easily. Further, we find that the $60 \mathrm{~nm}$ thickness of gold layer is not suitable for a precise and accurate sensing due to the low loss of the subpeaks in the longer wavelength.

\section{CONCLUSION}

In this paper, we have investigated a common 6-fold Dshaped hexagonal photonic crystal for a wider refractive index detection range. The numerical results show that the proposed sensor could achieve a maximum sensitivity of $7900 \mathrm{~nm} / \mathrm{RIU}$ $(\mathrm{RI}=1.33$ to 1.41$), 5300 \mathrm{~nm} / \mathrm{RIU}(\mathrm{RI}=1.33$ to 1.45$)$ with a thick analyte binding layer of $1500 \mathrm{~nm}$ and a thin analyte binding layer of $500 \mathrm{~nm}$, respectively. Further, we have observed two different types of SPRs named as 'dielectriclike' SPR with low loss and 'plasmon-like' SPR with high loss. Furthermore, in the 'plasmon-like' SPR, the normal phase matching condition takes place which demands the imaginary 
parts of $n_{\text {eff }}$ to be equal, whereas for 'dielectric-like' SPR, the real parts of $n_{\text {eff }}$ to be equal. According to the confinement loss spectra of various analytes, we have noticed that there are three main factors that turn out to be the limitation on the sensor's detection range. On one hand, as the secondary surface plasmon resonance is a 'plasmon-like' SPR type with high loss, in the sensing process, the main resonance sensing curve shifts close to the sub-peak resonance wavelength. Therefore, the sensing curve becomes blunt and undetectable. On the other hand, due to the existence of sub-peaks in the longer wavelength region, the resonant peaks overlap and affect the sensing performance sensor. Moreover, the dualpeak characteristic is also against the deep single-resonantpeak measurement requirement and leads to inaccurate and erroneous detection. By reducing the thickness of analyte's binding layer, it is possible to minimize the impact of the second SPR on the sensing range, resulting in the extension of the sensing range but slightly reduced sensitivity. As the D-shaped hexagonal PCF-SPR sensor is one of the most promising and fabricable SPR sensors, we envisage that, under the proper control during the sensing process, it could be an accurate and precise sensing device for a wide range of analyte refractive index detection.

\section{REFERENCES}

[1] R. Jorgenson and S. Yee, "Control of the dynamic range and sensitivity of a surface plasmon resonance based fiber optic sensor," Sensors and Actuators A: Physical, vol. 43, no. 1, pp. $44-48$, 1994. [Online]. Available: http://www.sciencedirect.com/science/article/ pii/092442479300661M

[2] N. Luan, R. Wang, W. Lv, and J. Yao, "Surface plasmon resonance sensor based on d-shaped microstructured optical fiber with hollow core," Opt. Express, vol. 23, no. 7, pp. 8576-8582, Apr 2015. [Online]. Available: http://www.opticsexpress.org/abstract.cfm? URI=oe-23-7-8576

[3] Y. Zhao, Z. qun Deng, and J. Li, "Photonic crystal fiber based surface plasmon resonance chemical sensors," Sensors and Actuators B: Chemical, vol. 202, pp. 557 - 567, 2014.

[4] E. Kretschmann and H. Raether, "Notizen: Radiative decay of non radiative surface plasmons excited by light," Zeitschrift für Naturforschung A, vol. 23, pp. 2135-2136, 1968

[5] A. K. Sharma and B. D. Gupta, "Fibre-optic sensor based on surface plasmon resonance with ag-au alloy nanoparticle films," Nanotechnology, vol. 17, no. 1, pp. 124-131, dec 2005. [Online]. Available: https://doi.org/10.1088\%2F0957-4484\%2F17\%2F1\%2F020

[6] Z. Liu, Y. Wei, Y. Zhang, Y. Zhang, E. Zhao, J. Yang, and L. Yuan, "Twin-core fiber spr sensor," Opt. Lett., vol. 40, no. 12, pp. 2826-2829, Jun 2015. [Online]. Available: http://ol.osa.org/abstract. cfm?URI=ol-40-12-2826

[7] Y.-C. Kim, W. Peng, S. Banerji, and K. S. Booksh, "Tapered fiber optic surface plasmon resonance sensor for analyses of vapor and liquid phases," Opt. Lett., vol. 30, no. 17, pp. 2218-2220, Sep 2005. [Online]. Available: http://ol.osa.org/abstract.cfm?URI=ol-30-17-2218

[8] M.-H. Chiu, S.-F. Wang, and R.-S. Chang, "D-type fiber biosensor based on surface-plasmon resonance technology and heterodyne interferometry," Opt. Lett., vol. 30, no. 3, pp. 233-235, Feb 2005. [Online]. Available: http://ol.osa.org/abstract.cfm?URI=ol-30-3-233

[9] T. Guo, F. Liu, B.-O. Guan, and J. Albert, "[invited] tilted fiber grating mechanical and biochemical sensors," Optics \& Laser Technology, vol. 78, pp. 19 - 33, 2016. [Online]. Available: http://www.sciencedirect.com/science/article/pii/S0030399215303893

[10] A. E. Khalil, A. H. El-Saeed, M. A. Ibrahim, M. E. Hashish, M. R. Abdelmonem, M. F. O. Hameed, M. Y. Azab, and S. S. A. Obayya, "Highly sensitive photonic crystal fiber biosensor based on titanium nitride," Optical and Quantum Electronics, vol. 50, p. 158, 2018.
[11] S. Chu, K. Nakkeeran, A. M. Abobaker, S. S. Aphale, P. R. Babu, and K. Senthilnathan, "Design and analysis of surface-plasmon-resonancebased photonic quasi-crystal fiber biosensor for high-refractive-index liquid analytes," IEEE Journal of Selected Topics in Quantum Electronics, vol. 25, no. 2, pp. 1-9, March 2019.

[12] B. Shuai, L. Xia, Y. Zhang, and D. Liu, "A multi-core holey fiber based plasmonic sensor with large detection range and high linearity," Opt. Express, vol. 20, no. 6, pp. 5974-5986, Mar 2012. [Online]. Available: http://www.opticsexpress.org/abstract.cfm?URI=oe-20-6-5974

[13] X. Yu, Y. Zhang, S. Pan, P. Shum, M. Yan, Y. Leviatan, and C. Li, "A selectively coated photonic crystal fiber based surface plasmon resonance sensor," Journal of Optics, vol. 12, no. 1, p. 015005 , dec 2009. [Online]. Available: https://doi.org/10.1088\%2F2040-8978\% 2F12\%2F1\%2F015005

[14] M. R. Hasan, S. Akter, A. A. Rifat, S. Rana, K. Ahmed, R. Ahmed, H. Subbaraman, and D. Abbott, "Spiral photonic crystal fiber-based dual-polarized surface plasmon resonance biosensor," IEEE Sensors Journal, vol. 18, no. 1, pp. 133-140, Jan 2018.

[15] M. Tian, P. Lu, L. Chen, C. Lv, and D. Liu, "All-solid dshaped photonic fiber sensor based on surface plasmon resonance," Optics Communications, vol. 285, no. 6, pp. 1550 - 1554 , 2012. [Online]. Available: http://www.sciencedirect.com/science/article/ pii/S0030401811013447

[16] Y. Chen, Q. Xie, X. Li, H. Zhou, X. Hong, and Y. Geng, "Experimental realization of d-shaped photonic crystal fiber SPR sensor," Journal of Physics D: Applied Physics, vol. 50, no. 2, p. 025101, nov 2016. [Online]. Available: https://doi.org/10.1088\% 2F1361-6463\%2F50\%2F2\%2F025101

[17] D. Monzón-Hernández, J. Villatoro, D. Talavera, and D. LunaMoreno, "Optical-fiber surface-plasmon resonance sensor with multiple resonance peaks," Appl. Opt., vol. 43, no. 6, pp. 1216-1220, Feb 2004. [Online]. Available: http://ao.osa.org/abstract.cfm?URI=ao-43-6-1216

[18] E. K. Akowuah, T. Gorman, H. Ademgil, S. Haxha, G. K. Robinson, and J. V. Oliver, "Numerical analysis of a photonic crystal fiber for biosensing applications," IEEE Journal of Quantum Electronics, vol. 48, no. 11, pp. 1403-1410, Nov 2012.

[19] A. Hassani and M. Skorobogatiy, "Design criteria for microstructuredoptical-fiber-based surface-plasmon-resonance sensors," J. Opt. Soc. Am. $B$, vol. 24, pp. 1423-1429, 2007.

[20] P. Russell, "Photonic crystal fibers," Science, vol. 299, pp. 358-362, 2003.

[21] N. Ashok and W. Shin, "Effective d-shape fiber with air hole assistant design for birefringence analysis," Optik, vol. 162, pp. 27 - 34, 2018.

[22] N. Zhang, K. Li, Y. Cui, Z. Wu, P. P. Shum, J.-L. Auguste, X. Q. Dinh, G. Humbert, and L. Wei, "Ultra-sensitive chemical and biological analysis via specialty fibers with built-in microstructured optofluidic channels," Lab Chip, vol. 18, pp. 655-661, 2018.

[23] J.Boehm, A.François, H.Ebendorff-Heidepriem, and T.M.Monro, "Chemical deposition of silver for the fabrication of surface plasmon microstructured optical fibre sensors," Plasmonics, vol. 6, pp. 133-136, 2011.

[24] X. Yang, Y. Lu, L. Duan, B. Liu, and J. Yao, "Temperature sensor based on hollow fiber filled with graphene-ag composite nanowire and liquid," Plasmonics, vol. 12, pp. 1805-1811, 2017.

[25] P. Leonard, S. Hearty, J. Quinn, and R. OKennedy, "A generic approach for the detection of whole listeria monocytogenes cells in contaminated samples using surface plasmon resonance," Biosensors and Bioelectronics, vol. 19, pp. 1331 - 1335, 2004.

[26] X. Chen, L. Xia, and C. Li, "Surface plasmon resonance sensor based on a novel d-shaped photonic crystal fiber for low refractive index detection," IEEE Photonics Journal, vol. 10, pp. 1-9, 2018.

[27] R. by: Amit Asopa and Z. H. Bajwa, "Handbook of acute pain management," Journal of Pain \& Palliative Care Pharmacotherapy, vol. 25, no. 4, pp. 394-394, 2011. [Online]. Available: https: //doi.org/10.3109/15360288.2011.620691

[28] S. Ke, B. Wang, H. Huang, H. Long, K. Wang, and P. Lu, "Plasmonic absorption enhancement in periodic cross-shaped graphene arrays," Opt. Express, vol. 23, no. 7, pp. 8888-8900, Apr 2015. [Online]. Available: http://www.opticsexpress.org/abstract.cfm?URI=oe-23-7-8888

[29] S. Gong, T. Zhao, M. Sanderson, M. Hu, R. Zhong, X. Chen, P. Zhang, C. Zhang, and S. Liu, "Transformation of surface plasmon polaritons to radiation in graphene in terahertz regime," Applied Physics Letters, vol. 106, no. 22, p. 223107, 2015. [Online]. Available: https://doi.org/10.1063/1.4922261

[30] J. N. Dash and R. Jha, "Spr biosensor based on polymer pcf coated with conducting metal oxide," IEEE Photonics Technology Letters, vol. 26 , pp. 595-598, 2014. 
[31] G. Wang, S. Li, G. An, X. Wang, Y. Zhao, W. Zhang, and H. Chen, "Highly sensitive d-shaped photonic crystal fiber biological sensors based on surface plasmon resonance," Optical and Quantum Electronics, vol. 48, no. 1, p. 46, Dec 2015. [Online]. Available: https://doi.org/10.1007/s11082-015-0346-4

[32] G. An, S. Li, X. Yan, X. Zhang, Z. Yuan, H. Wang, Y. Zhang, $X$. Hao, Y. Shao, and Z. Han, "Extra-broad photonic crystal fiber refractive index sensor based on surface plasmon resonance," Plasmonics, vol. 12, no. 2, pp. 465-471, Apr 2017. [Online]. Available: https://doi.org/10.1007/s11468-016-0286-2

[33] Z. Zhang, Y. Shi, B. Bian, and J. Lu, "Dependence of leaky mode coupling on loss in photonic crystal fiber with hybrid cladding," Opt. Express, vol. 16, no. 3, pp. 1915-1922, Feb 2008. [Online]. Available: http://www.opticsexpress.org/abstract.cfm?URI=oe-16-3-1915

[34] H. Lu, X. Liu, and D. Mao, "Plasmonic analog of electromagnetically induced transparency in multi-nanoresonator-coupled waveguide systems," Phys. Rev. A, vol. 85, p. 053803, 2012.

[35] W. DiPippo, B. J. Lee, and K. Park, "Design analysis of dopedsilicon surface plasmon resonance immunosensors in mid-infrared range," Opt. Express, vol. 18, no. 18, pp. 19396-19406, Aug 2010. [Online]. Available: http://www.opticsexpress.org/abstract.cfm? URI=oe-18-18-19396

[36] M. S. A. Gandhi, S. Chu, K. Senthilnathan, P. R. Babu, K. Nakkeeran, and $\mathrm{Q}$. $\mathrm{Li}$, "Recent advances in plasmonic sensor-based fiber optic probes for biological applications," Applied Sciences, vol. 9, no. 5, 2019. [Online]. Available: https://www.mdpi.com/2076-3417/9/5/949

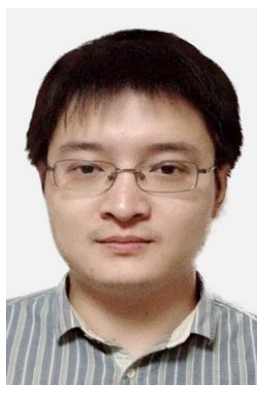

Suoda Chu received the B.Eng. degree (Communication Engineering) from Nanjing University Jinling College, Nanjing, China, in 2010, the MSc degree (Microwave and Wireless Communication Engineering) from Cardiff University, Cardiff, UK, in 2014, and is currently working toward his Ph.D degree in school of engineering, University of Aberdeen, Aberdeen, UK.

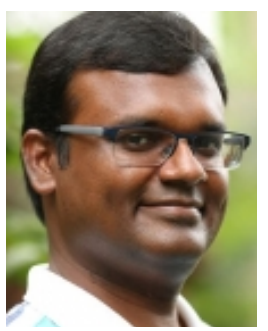

K. Nakkeeran (Ph.D., CEng, SMIEEE, SMOSA, MIET) received the B.Eng. degree from the Coimbatore Institute of Technology, Coimbatore, India, in 1993, and the M.Tech. and Ph.D. degrees from Anna University, Chennai, India, in 1995 and 1998, respectively. In 1999, he joined the Institute of Mathematical Sciences, Chennai, where he was a Post-Doctoral Fellow for ten months. In 1999, he became a Research Associate with the Department of Physics, University of Burgundy, Dijon, France. In 2002, he became a Post-Doctoral Fellow with the Department of Electronic and Information Engineering, The Hong Kong Polytechnic University. In 2005, he joined the School of Engineering, University of Aberdeen, Aberdeen, U.K., where he has been a Senior Lecturer since 2011. His research interests include solitons, fiber lasers, fiber sensors, modeling and simulations of optical devices, long-haul optical fiber communications, and nonlinear science.

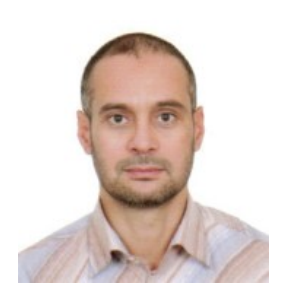

Abdosllam M. Aboboaker received the High Diploma in Electronic Engineering- Higher Institute of Electricity, Zliten, Libya. (September 1995), M.Eng. in Engineering (communication and computer)- National University of Malaysia (UKM) Bangi Selangor, Malaysia.(November 2003), and $\mathrm{Ph} . \mathrm{D}$. in Engineering University of Aberdeen, Aberdeen, UK (November 2009), he joined the College Of Electronic Technology Bani Walid Libya as faculty member in 2010 , he became a dean of the college in 2011, he appointed as President of the Constituent Committee for the establishment of the Supreme Council of Technical and Vocational Education, Ministry of higher Education Libya in May 2012, His research interests include solitons, fiber lasers, long-haul optical fiber communications, and nonlinear science modeling and simulations of optical devices,. Dr. Abdosllam is a member of the Optical Society of America and The Institute of Electrical and Electronics Engineers (IEEE).

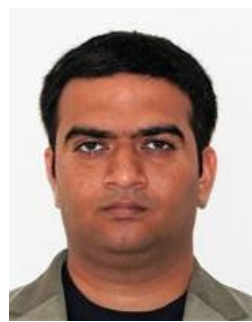

Sumeet S. Aphale (Ph.D., CEng, SMIEEE, MIET) is a Senior Lecturer at the School of Engineering, University of Aberdeen. His has earned the B.E. (University of Pune, India 1999), M.S. (University of Wyoming, USA 2003) and Ph.D. (University of Wyoming, USA, 2005) degrees, all in Electrical Engineering with a focus on Robotics and Control. He has held post-doctoral research positions at the ARC Centre of Excellence for Complex Dynamic Systems and Control, University of Newcastle (2006-08) and at the Centre for Applied Dynamics Research, University of Aberdeen (2008-09). His research interests include nanopositioning, drill-string dynamics, vibration control, flexible manipulators, Gough-Stewart platforms, biomedical devices and fibre-optic sensors; and has published over 60 papers in peer-reviewed journals and conferences. He is an Associate Editor for the IEEE Control Systems Societys Conference Editorial Board. He is also serves as an Associate Editor for Shock and Vibration as well as for Frontiers of Mechanical Engineering (Mechatronics Section).

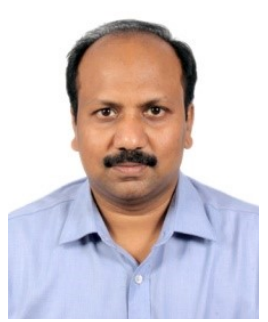

S. Sivabalan received his B. Tech. degree in Control \& Instrumentation Engineering, June 2000, M.Tech. degree in Laser and electro-optical engineering from the College of Engineering, Anna University, Chennai, India in Dec. 2002, and the Ph.D. degree from VIT University, Vellore, India, in July 2013. From 2013 to 2016, he was an Associate Professor at the Department of Electrical Engineering, Vellore Institute of Technology. Since 2017, he has been holding the position of Professor in the School of Electrical Engineering. He was a visiting researcher of Bilkent University, Ankara, Turkey. His research interests include ultrafast fiber laser, fiber optic sensor, microstructured fibers, visible light communication and nonlinear optical imaging.

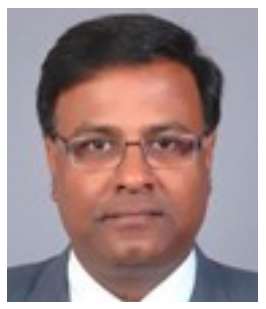

P. Ramesh Babu is a Professor at VIT, Vellore, India. He earned M.Sc., M.Phil., and Ph.D. degrees in Physics from University of Madras, Chennai. His areas of research include fiber Bragg gratings, optical fiber communications, photonic crystal fibers, pulse compression, metamaterials and Bose-Einstein condensation.

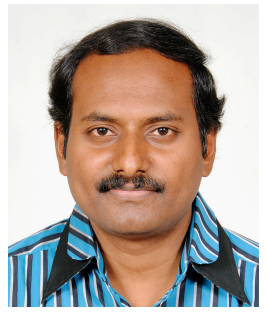

K. Senthilnathan is a Professor at VIT, Vellore. He earned M.Sc and M.Phil. degrees in Physics from University of Madras, and Ph.D. degree from Anna University, Chennai. His areas of research include optical fibers, fiber Bragg gratings, photonic crystal fibers, pulse compression, Bose-Einstein condensation and metamaterials. 